\title{
Medizin in (Latein)Amerika vor der Conquista Teil II: Mesoamerikanische Kulturen
}

\author{
Reto Brignoli1, Reinhard Saller2 \\ 1 Facharzt für klinische Pharmakologie, CH-Rüti; 2 UniversitätsSpital Zürich, Institut für Naturheilkunde, CH-Zürich
}

n Teil I dieses Beitrags [1] haben wir zunächst eine Einführung in die Volksmedizin (Latein)Amerikas in der Zeit vor der Conquista (d.h. der Eroberung durch die Europäer) gegeben und im Anschluss über die Medizin der Andenregion berichtet.

Der vorliegende Teil II behandelt die Medizin in den Mesoamerikanischen Kulturen.

\section{Das Land und sein Umfeld}

Einer der stärksten Vulkanausbrüche des letzten Jahrtausends war der Ausbruch des Tambora in Indonesien 1815; der Vulkan stiess rund $100 \mathrm{Ku}-$ bikkilometer Magma, Staub und Asche aus und katapultierte 400 Millionen Tonnen Schwefelgase bis zu 44 Kilometer hoch in die Atmosphäre. Das folgende Jahr ging als „Jahr ohne Sommer“ in die Geschichte ein - sowohl in Europa als auch in Nordamerika war es kalt, schneite bis in den Sommer. In der Schweiz führte dies zur letzten grossen Hungersnot. Es wird wissenschaftlich kaum bestritten, dass die starke Abkühlung 1816 eine Folge dieses Vulkanausbruchs war [2]. Im Juni und Juli gab es in den USA jede Nacht Frost. In New York und Neuengland fiel bis $\mathrm{zu}$ einem Meter Schnee. In Deutschland kam es zu schweren Unwettern; zahlreiche Flüsse traten über die Ufer. In der Schweiz schneite es jeden Monat mindestens einmal bis auf $800 \mathrm{~m}$ Meereshöhe. Im August setzte auch in Europa der Frost ein, die Folge waren katastrophale Missernten. Im Frühjahr 1817 stiegen die Getreidepreise auf das Zehnfache, Hungersnöte brachen unter der Land-
Die präkolumbianischen Einwohner waren enorm zerstörerischen umweltbedingten Einflüssen im tektonisch-vulkanischen „Feuerring" ausgesetzt. Darin dürfte der Ursprung der zyklischen Weltvorstellung mit der immer wiederkehrenden Drohung eines Weltuntergangs liegen, dem mit Menschenopfern, blutigen Selbstkasteiungen, rituellem Kannibalismus zu begegnen war. Es gibt zahlreiche Hinweise auf einen mongolischen Ursprung der Amerikanischen Urbevölkerung. Die erste Hochkultur Amerikas, die Olmekenkultur, entstand vor ca. 3000 Jahren im Osten Mexikos. Die späteren Kulturen Zentralamerikas hatten diverse gemeinsame Merkmale. Der Säuberungswut der Inquisition entgingen nur wenige Schriftstücke die z.T. als Kopie bis heute erhalten sind. Erhaltene Codices gibt es von den Maya, den Azteken und den Mixteken. Der in medizinischen Angelegenheiten meist zitierte Codex Florentinus stammt aus der spanischen Kolonialepoche. Die Gesundheit wird als das Resultat des Gleichgewichts der Wärme-Kälte-Polarität betrachtet. Geht dieses Gleichgewicht verloren, so entsteht eine krankheitverursachende Störung in der Beziehung zwischen Körper und Kosmos. Der Kakao diente nicht nur als Genussmittel, sondern auch als Zahlungsmittel und als Therapeutikum z.B. bei Depression. Als Medizinalpflanzen galten z.B. die Chilischoten, die spasmolytischen Casimiroa spp., die Oxytocinartige Montanoa tomentosa, die laxativ wirkende Cáscara sagrada, der Agavensaft. Der Erfahrungsschatz aus dieser Region liefert immer wieder neue pharmakologische Ansätze; ethnomedizinische Informationen werden weltweit gesammelt und in verschiedenen Datenbanken zugänglich gemacht.

Schlüsselwörter: Medizinalpflanzen, Ethnobotanik, Traditionelle Medizin, Paleopathologie, Mayas, Azteken, Zapoteken

\section{Pre-Columbian Medicine in (Latin)America. Part 2: Mesoamerican Cultures}

The pre-Columbian inhabitants of central America were exposed to tremendously destructive influences emanating from the surrounding tectonic and volcanic, ring of fire'. One may hypothesize that herein lies the origin of their cyclic vision of the world, with the ever-returning imminence of an apocalypse which had to be avoided with human sacrifices, bloody self-chastening and ritual cannibalism. The indigenous American population appears to be of Mongolic origin. The first developed civilization in America was that of the Olmecs, which originated in the east of today's Mexico about 3000 years ago. Later central American civilizations shared a large number of characteristics. Very few written texts escaped the depurative wrath of the holy inquisition; part of the saved texts have been preserved as copies until today. There are known Codices from the Mayas, the Aztecs and the Mixtecs. The Codex Florentinus, which is the most quoted codex when it comes to medical issues, stems from the early colonial period. In the view of central American cultures, health results from the equilibrium of the polarity between hot and cold. If this equilibrium is disturbed, it will generate a disease-causing alteration in the relation between the body and the cosmos. Cocoa was not only important as a drink, but also as currency and as a medicine, e.g. against depression. The long list of medicinal plants contains chili pepper, the spasmolytic Casimiroa spp., the oxytocinic Montanoa tomentosa, the laxative Cascara sagrada as well as the juice of the agave. Time and again, the vast treasure of experience in this region provides new pharmacological approaches; ethnomedical information are gathered worldwide and are accessible through various data-bases.

Key Words: medicinal plants, ethnobotany, traditional medicine, Paleopathology, Maya, Aztecs, Zapotecs 


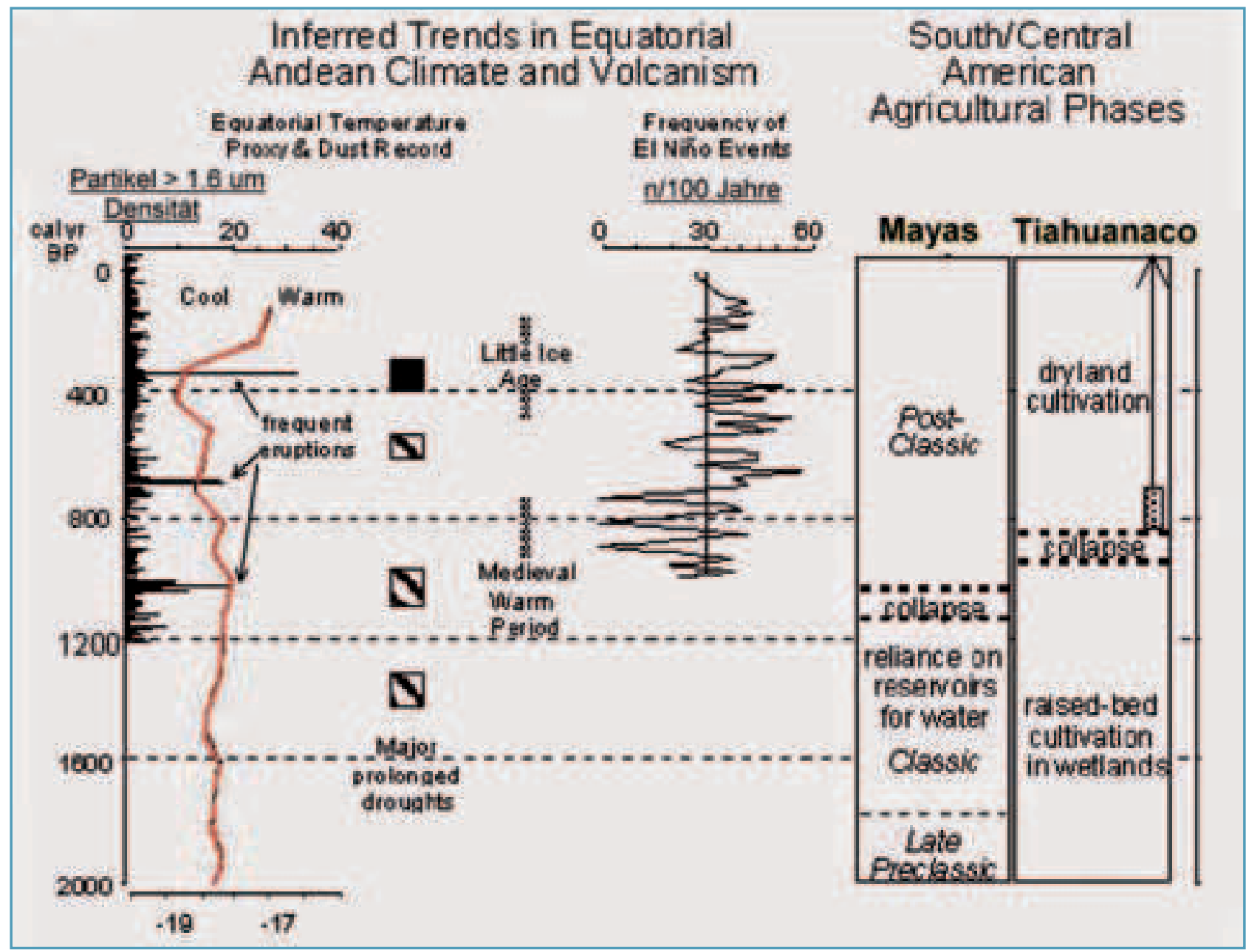

Abb. 1. Zusammenhang zwischen Wärmezyklen, Vulkanaktivität und ,El Niño' einerseits und Perioden zentral- und südamerikanischer Kulturen.

bevölkerung aus. In St. Gallen wurden 6000 Hungertote gezählt, Appenzell verlor 6\% der Bevölkerung. Der Chronist R. ZOLLIKOFER schrieb 1818: "Die wohlthätigen Sennen hatten bis ungefähr Anfangs März nur sehr wenig Schotten oder Molken; daher man immer mehr zu den elendesten, eckelhaftesten Speisen Zuflucht nehmen musste, und was ehedem Schweinen nicht wäre vorgeworfen worden, das genossen nun diese Tausend Hungrigen noch als köstliche Speise: «Oehmd oder Grummet auf dem Ofen gedörrt» - schreibt mir der Tit. Landammann Bischoffberger- «dann zu Mehlstaub zerrieben mit Schotten gekocht, wurden geniessbarer Brey; gedörrte Erdäpfelhülsen mussten mit und ohne Grüsche (Kleie) mit Wasser abgekocht zur Suppe dienen. Schindlinge, zer-

1 http://www.geo.mtu.edu/volcanoes/world.html mahlne Knochen, Pferdefleisch, Zumehl, Leim, Blut, Häute von Thieren, hielten die Hungrigen für gute Nahrungsmittel; Hunde und Katzen waren für sie Leckerbissen; und braunes Heu abgesotten, dann den Absud gesalzen, fanden unsere Armen als schmackhafte Suppe; usw., usw..»" [3].

Das ist zwar nicht Zentralamerika, vermittelt aber doch eine lebhafte Vorstellung über die umweltbedingten Einflüsse, denen die präkolumbianischen Einwohner in dem vulkanreichen, erdbebengeschüttelten Zentralamerika ausgesetzt waren. Zentralamerika befindet sich im tektonischvulkanischen „Feuerring”; die bedeutendsten Vulkane ${ }^{1}$ sind: in Mexico der Popocatepetl und Paricutin, in Guatemala der Santa María, Agua, Pacaya del Fuego, Tacaná und der Cerro Quemado; in Costa Rica der Arenal und Rincon de la Vieja, in El Salvador der
Coatepeque, Ilopango, Izalco, San Miguel, San Salvador, San Vicente und Santa Ana; in Nicaragua der Cerro Negro. Paleoklimatologische Studien belegen die massiven akuten Veränderungen durch Erdbeben und Vulkanausbrüche, die immer wieder vernichtend auf Zentralamerika einwirkten [4,5] (Abb. 1 und 2). Ob wohl hierin die Wurzel der Angst und der Grausamkeit der mesoamerikanischen Kulturen, mit Menschenopfer, blutiger Selbstkasteiung, rituellem Kannibalismus usw. liegt? Ob wohl darin der Ursprung der zyklischen Weltvorstellung mit der immer wiederkehrenden Drohung eines Weltuntergangs zu suchen ist?

Seit 2000 v. Chr. errichteten indianische Brandrodungsbauern ihre ersten Siedlungen im Tiefland von Guatemala und Belize, bevorzugt in der Nähe von flachen Bodensenken (spanisch: Bajo). 


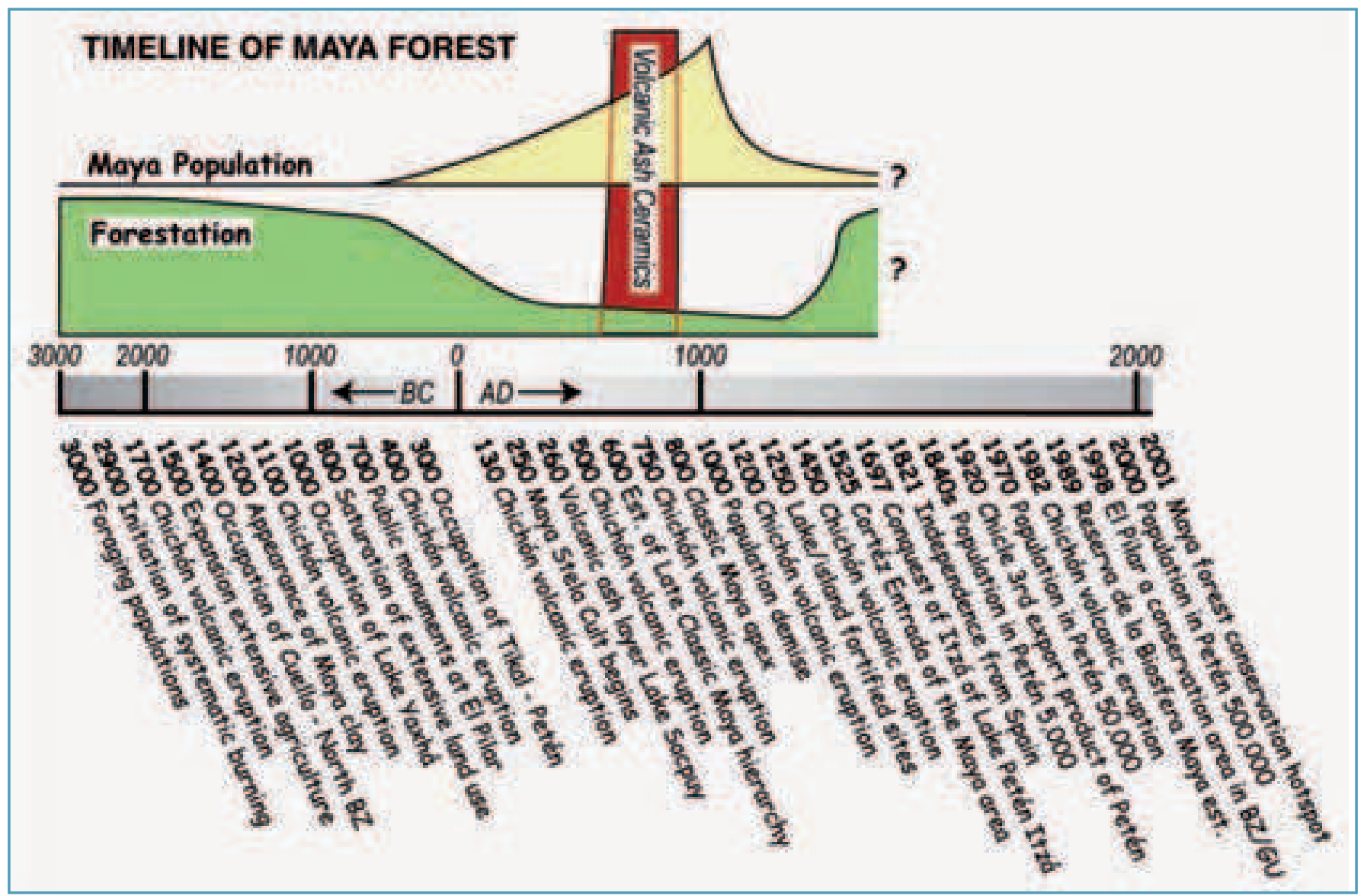

Abb. 2. Zusammenhang zwischen Forestation und Vulkanaktivität einerseits und Perioden der Maya Kultur

Die sumpfigen Karstmulden im Kalksteinboden werden heute nur noch zwischen Juli und November bewässert. Diese ursprünglich hydrologisch stabilen Ökosysteme stellten vermutlich ein attraktiveres Siedlungsgebiet dar als die Sümpfe, die sich heute während einiger Monate bilden. Um 100 n. Chr. war das Oberflächenwasser während des grössten Teils des Jahres verschwunden, eine Tonschicht verschloss die tiefer gelegenen Torflagen. Die Wissenschaftler machen für diese Umweltschäden den exzessiven Brandrodungsbau der Maya in Verbindung mit einem Klimawandel verantwortlich. Immer grössere Waldflächen wurden zur Anlage von Feldern für die wachsende Bevölkerung gerodet, Steinbrüche für die Häuser der Städte zerfurchten das Land. Die Versumpfung der Bajos fällt in das Ende der präklassischen Zeit der Maya-Kultur (400 v. Chr. bis 150 n. Chr.), als viele Städte von ihren Bewohnern verlassen wurden. An anderen Orten (Zapoteken in
Oaxaca) gingen die Indianer zur Regulierung der Wasserversorgung über, indem sie Wasserbecken innerhalb ihrer Städte anlegten oder neue Quellen erschlossen. Spätere Trockenperioden, besonders zwischen 530-650 n. Chr. und 800-1000 n.Chr (Höhere Evaporationswerte $\mathrm{d} 18-\mathrm{O}_{2}$ und Gipsablagerungen in den Seen), und andere paleoklimatologisch belegte Umweltkatastrophen wie Vulkanaktivität (z.B. Partikel im Quelccayaeis im peruanischen Altiplano) waren die Hauptverantwortlichen für periodische Zusammenbrüche und den endgültigen Kollaps der Mayakulturstätten [6].

\section{Die Menschen}

\section{Ursprung, genetische Merkmale}

Die Gemeinsamkeiten der haplotypen mitochondrialen DNS weisen auf einen mongolischen Ursprung der amerikanischen Urbevölkerung hin [7]; die Völkerwanderungen über die Behring-
Landbrücke sollen in drei Perioden zwischen 30'000 und 7000 v.Chr. stattgefunden haben. Die Nord-Süd Wanderungen sollen relativ rasch gewesen sein, anschliessend waren die Bevölkerungen ziemlich stabil (d.h. Mutationen finden in einem geographisch umschriebenen Raum statt). Die Haplogruppen, mit A, B, C, und D gekennzeichnet, weisen ein Nord-Süd Gefälle auf (Haplogruppe A häufiger in Nordamerika, die restlichen häufiger in Südamerika) [8,9]. Indianer haben mongolische Körpermerkmale, und in früher Kindheit den Mongolenfleck; auch Gaumenspalten sind bei ihnen häufiger als bei anderen Bevölkerungsgruppen [10]. Nicht gänzlich auszuschliessen - aber von quantitativ untergeordneter Bedeutung - ist, dass auch über den Seeweg Einwanderer den Weg nach Amerika gefunden haben könnten, über den Süd- und/oder Nordpazifik oder aus Europa (wie Thor Heyerdahl in seinen verschiedenen Expeditionen nahe gelegt hat). Ein 
verbreitetes Merkmal ist das Laktasedefizit (LAC*P-Gen) sowie - bei der heutigen Bevölkerung - Obesität, Diabetes mellitus, Gallensalz- und Gallenblasenkrankheiten. Auf der anderen Seite haben sie ein geringeres Risiko, kardiovaskuläre Erkrankungen zu entwickeln - trotz häufig erhöhten Triglyzeriden im Vergleich zu Mexikanern kaukasischen Ursprungs. Eine Untersuchung der Apolipoprotein E-Gen (APO E)- Polymorphismen zeigte, dass die vorwiegend amerindische Bevölkerung eine reduzierte Häufigkeit der APO E-2 und APO E-4-Varianten aufweist (diese sollen für die Entwicklung von Atherosklerose und Hyperlipidemien bei Kaukasiern von Bedeutung sein). Auch die Quasiabwesenheit des ACE D/D-Genotyps dürfte eine kardiovaskulär protektive Wirkung haben, wie auch an Yanomami ,Indios', die praktisch nie an Hypertonie leiden, gezeigt wurde [11].

\section{Die kulturellen Hochburgen}

Vor ca. 3000 Jahren entstand die erste Hochkultur Amerikas, die Olmekenkultur im tropische Osten Mexikos (San Lorenzo Tenochtitlán, La Venta, Cerro de las Mesas und Tres Zapotes in der Gegend des heutigen Tabasco und Veracruz). Durch die jährlichen Überschwemmungen der Flüsse wurde an deren Ufern Humus abgelagert wodurch 2 Ernten im Jahr möglich waren (Mais, Maniok, Kürbis, Bohnen und Süsskartoffeln). Die Olmeken gelten als die Schöpfer der Haupterfindungen des vorkolumbianischen Mesoamerikas so z. B. eine Schrift, die viel Ähnlichkeit mit der späteren Hieroglyphenschrift der Maya und Azteken hat, Ziffern (Vigesimalsystem) und wahrscheinlich auch die komplizierten Kalendersysteme. Der Name "Olmeken" stammt aus dem Nahuatl (Azteken) und bedeutet "das Volk des Kautschuklandes”.

Die erste indianische Hochkultur in Zentralmexiko wurde nach ihrem Hauptzentrum im Hochtal von Mexiko Teotihuacán benannt; es wurde vom Anfang des 1. bis zum 7. Jh. besiedelt. Teotihuacán war die Metropole eines Reiches, welches das der Azteken an Macht übertraf. Die Bewohner hinterliessen monumentale Bauwerke, wa-

Tab. 1. Gemeinsamkeiten Zentralamerikanischer Kulturen

Nach einem Plan gebaute Städte; monumentale Zeremonialplätze; zeremonielles Ballspiel

- Schrift mit Hieroglyphen und Piktogramme; geschrieben wurde vorwiegend auf einer Art Papier aus Agavenfasern oder Tierfellen.

- Bigesimales Rechnungssystem (inklusive die Null bei den Maya)

- Sternwarten sowie ein doppelter Kalender mit Jahresrechnungen zu 260 Tagen und zu 365 Tagen, die ineinandergreifend wie zwei Zahnräder liefen.

- Komplexe, meist theokratisch geprägte Regierungsformen

- Fortgeschrittene landwirtschaftliche Methoden, inklusive Bewässerungskanäle (i.B. Oaxaca) und sehr rege Handelstätigkeit - mit Artikeln von Kolumbien bis Mexico.

- Stark religiös geprägtes Alltagsleben; Schuld und Sühne mit Selbstkasteiung, (i.B. Aderlass bei den Azteken) usw.

- Das Rad war zwar bekannt in Kinderspielzeugen, wurde aber nie nutzbar gemacht; Lasten wurden - mangels Lasttieren - von Menschen transportiert.

ren gute Bodenbauern (Anbau von Mais, Bohnen, Baumwolle), sowie auch gute Weber, Töpfer, Maler und Schöpfer von schönen Mosaiken aus Halbedelsteinen. Leider wurden aus dieser hoch entwickelten Kultur keine Schriftzeichen übermittelt.

Zumindest chronologisch waren die Maya die Nachfolger der Olmeken. Es gab zwar kein einheitliches Maya-Reich sondern eine gemeinsame Kultur mit gemeinsamen Mythen, Schöpfungsgeschichte, Religion und Machtstrukturen in Stadtstaaten (Tab. 1). Anscheinend befanden sich diese Zentren in dauerndem Kriegszustand (die Gefangenen wurden dann in einem Opferritual gefoltert und getötet). Die Mayawelt kann man in 3 Zonen unterteilen: a) Die Zentralzone enthält im wesentlichen das Petén samt Belize. Es ist ein flaches, vom Tropenwald bedecktes Land. Dort haben sich die ältesten der grossen Mayastädte wie Tikal und Uaxactun gebildet (auch Bonampak, Yaxchilan, Piedras Negras und Palenque);

b) die Nordzone, die den grössten Teil der Halbinsel Yucatán umfasst, mit Städten wie Uxmál, Kabah, Labná und Chichén Itzá (siehe Tolteken), und

c) die Südzone mit dem Bergland Südguatemalas.

Es gab kein einheitliches MayaReich. Das Maya-Tiefland war in eine Reihe unabhängiger politischer Einheiten aufgeteilt, die den Stadtstaaten der Sumerer und Griechen ähnelten. Anscheinend befanden sich diese Zentren in dauerndem Kriegszustand, wobei bis zum 3. Jahrhundert anscheinend nicht territoriale Vergrösserungen, sondern die Gefangennahme hochrangiger Persönlichkeiten das Hauptziel der Kampfhandlungen war. Kriegführen und Gefangennehmen von Gegnern war für Maya-Könige enorm wichtig.

Die Azteken kamen erst im frühen 13. Jahrhundert vom Hochtal von Mexiko. Der exakte Zeitpunkt der Gründung Tenochtitláns ist nicht genau bekannt (ca. 1325-1370 n. Chr.). Die Azteken spalteten sich auf und ein Teil gründete Tlatelolco. Tenochtitlán war von Anfang an eine Doppelstadt: das eigentliche Tenochtitlán im Süden, das religiöses und politisches Machtzentrum war, und im Norden Tlatelolco, der Markt der Stadt. Um Spannungen zwischen beiden Städten zu vermeiden, war man bemüht, ein gemeinsames, zentrales Oberhaupt zu schaffen. Moctezuma I vergrösserte das Reich erheblich; nach seiner Herrschaft erstreckte sich der Machtbereich von Cuernavaca im Süden bis nach Xochitlalpan im Norden und von Osten, dem Land der Huaxteken, bis nach Tabasco. Diese Eroberungen brachten nicht nur Beute, sondern in den nachfolgenden Jahren auch Tribute in Form von Luxusartikeln, Nahrungsmitteln, Baumaterialien und Sklaven. Auch die Nachfolger von Moctezuma vergrösserten das Reich. Erst unter Moctezuma II. zeigte es sich, dass das Expansionsbestreben der Azteken an eine natürliche Grenze gestossen war. Die Staatsordnung der Azteken war auf Krieg ausgerichtet 
und man unterhielt gewaltige Heere. Dort, wo sich heute die Hauptstadt Mexicos befindet, war einmal Tenochtitlán; als Hernán Cortés 1519 einmarschierte, betrug die Einwohnerzahl wahrscheinlich ca. 100'000 Menschen. Die Medizin war unter den Azteken hoch entwickelt; sie unterschieden zwischen den „tepati”, die sich mit empirischer Medizin befassten, und den „ticitl”, die als Schamane und Magier agierten. Unter den „tepati” gab es die Chirurgen (texoxtl), die Internisten (tlamatepahtli), die Spezialisten für Aderlass (tecoania), die Geburtshelfer (temixiuitiani), und die Pharmazeuten (papiani und panamacani). Der menschliche Körper wurde von drei Grund prinzipien angetrieben, dem „tonalli” im Kopf, dem „teyolía” im Herz und dem „ihíyotl” in der Leber.

Weitere bedeutende Völker waren u.a. die Zapoteken, die Landwirtschaft betrieben und ein ausgefeiltes Bewässerungssystem entwickelten, welches z.T. noch heute funktioniert. Sie siedelten sich ca. 500 v. Chr. in Monte Albán (im Tal von Oaxaca) an. Es war das kulturelle, politische und militärische Zentrum. Zwischen 500-200 v.Chr, wurde der Berggipfel plateauartig eingeebnet. In Monte Albán findet man Steinplatten aus dieser Zeit, die Abbildungen so genannter “Tänzer” (span. Danzantes) zeigen (Abb. 3). Es sind menschliche Figuren in diversen eigenartigen Stellungen, die verschiedene Krankheiten sowie die Geburt und das Alter darstellen sollen; z.T. sind Gebärmutter und Eileiter aufgezeichnet. Möglicherweise befand sich in Monte Albán die erste Medizinschule Zentralamerikas [12]. Die Zapoteken hatten auch eine eigene Hieroglyphenschrift, die auf diesen “Tänzer"-Steinplatten zu finden ist. Zwischen 1000-1500 n. Chr. wurden die Zapoteken von den Mixteken aus Monte Albán vertrieben. Daraufhin wurde Mitla die neue Hauptstadt. Wie auch andere Stämme, deformierten sie ihre Schädel, feilten ihre Zähne spitz und färbten sie (Monte Albán IIIA: 250 -300 n.Chr.) (Abb. 4) oder versahen sie mit Jade oder Goldeinlagen (vergl. $[13,14])$. Im Gegensatz zu den Maya, wurden hier auch Trepanationen ab 300 n. Chr. durchgeführt und teilweise

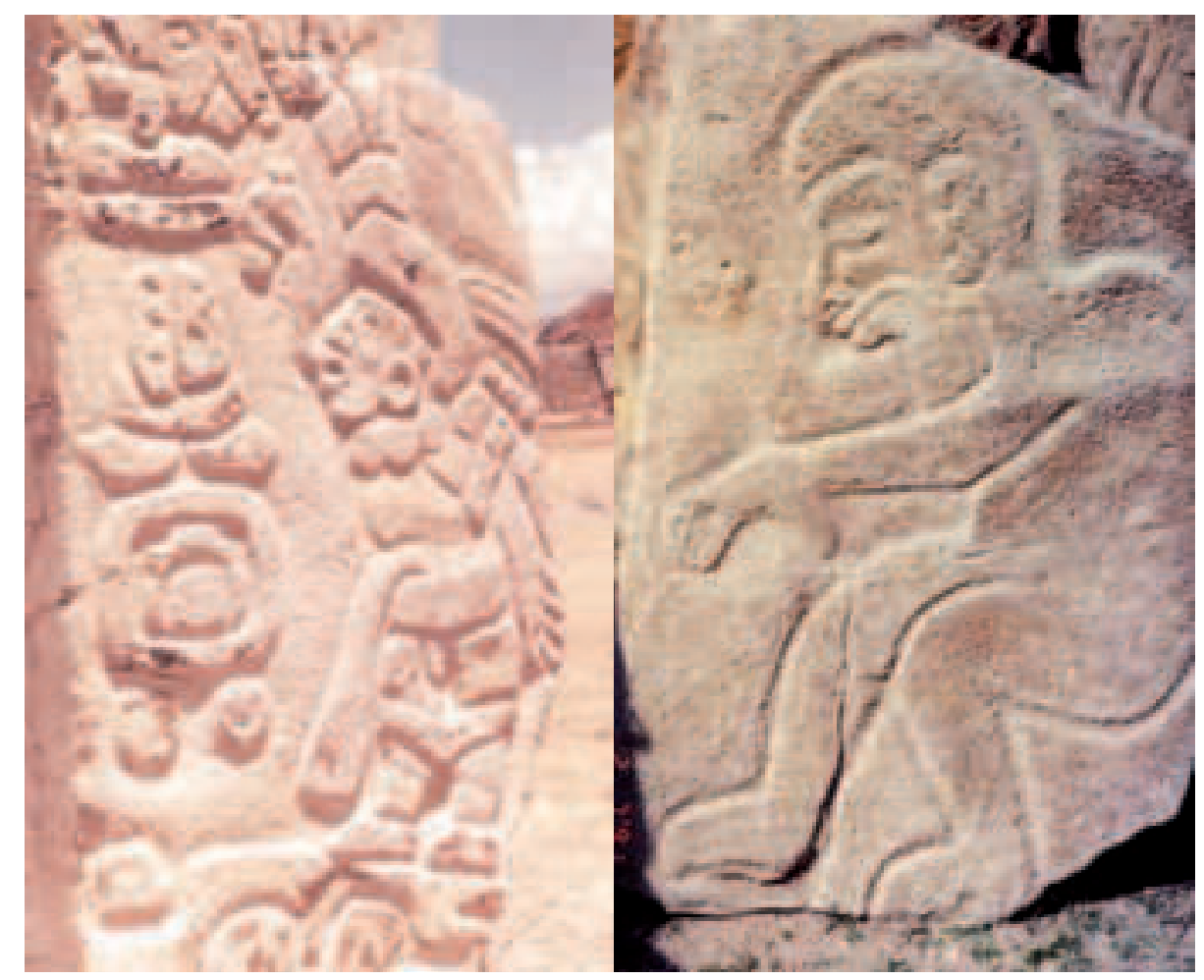

Abb. 3. Danzantes (Monte Albán). Man dachte ursprünglich, es wären Tänzer (d.h. Danzantes), später hielt man sie für Gefangene, und heute glaubt man, dass sie Kranke mit ihren Krankheiten darstellen könnten.

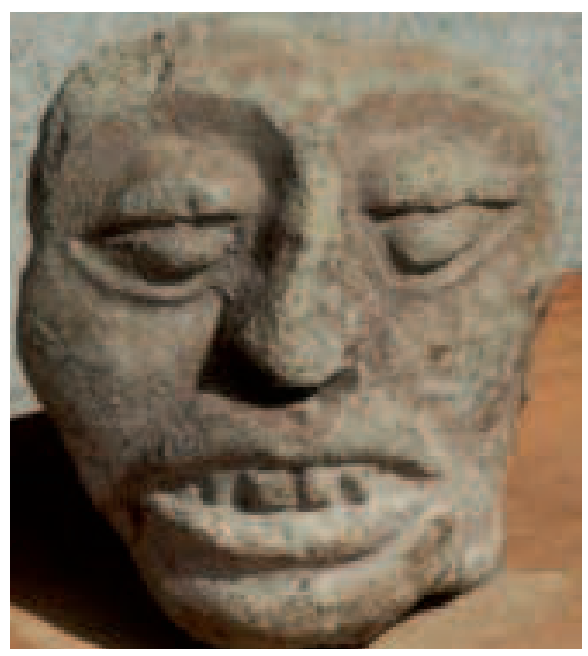

Abb. 4. Die Zapoteken deformierten ihre Schädel und feilten ihre Zähne durch Abbrasion mit Sand oder Quartzpulver (Monte Albán).

auch von den Patienten überlebt [15].

Die Hauptstadt der Tolteken war Tollan (bzw. Tula), das Zentrum einer Konföderation der ältesten mexikanischen Städte. Gegen Ende der klassischen Zeit siedelten sich die Chichimeken in Tula an und vereinigten sich mit dem dort lebenden Volk der Tolteken. Dieses Mischvolk schuf eine neue Kultur, die von mittelamerikanischen Zentren beeinflusst war. Der berühmteste und zugleich letzte Herrscher der ersten toltekischen Dynastie war Quetzalcoatl, der später zur Gottheit erhoben worden war. Durch einen Bürgerkrieg wurde er vertrieben. Mit ihm zusammen verliessen zahlreiche Gruppen von Tolteken das Land und gingen in den Süden Mexikos und beeinflussten kulturell die dort lebenden Mixteken, während andere die Halbinsel Yucatán erreichten, die von den Maya besiedelt wurde, was zu einer neue Mischkultur führte (u.a. Chichén Itzá, Tulum, Uxmál)

Die Huaxteken, im Norden des totonakischen Gebiets, hatten eine ähnliche Sprache wie die Maya, teilten aber sonst kein weiteres kulturelles Merkmal mit den Maya. Wahrscheinlich haben sich die Huaxteken von den Bewohnern des Petén und Yucatán im Laufe einer Wanderung getrennt. Die Huaxteken waren als grausames, aber sehr mutiges und begabtes Kriegervolk bekannt. Selbst die Azteken und später die Spanier haben die Huaxteken niemals völlig unterwerfen können. 


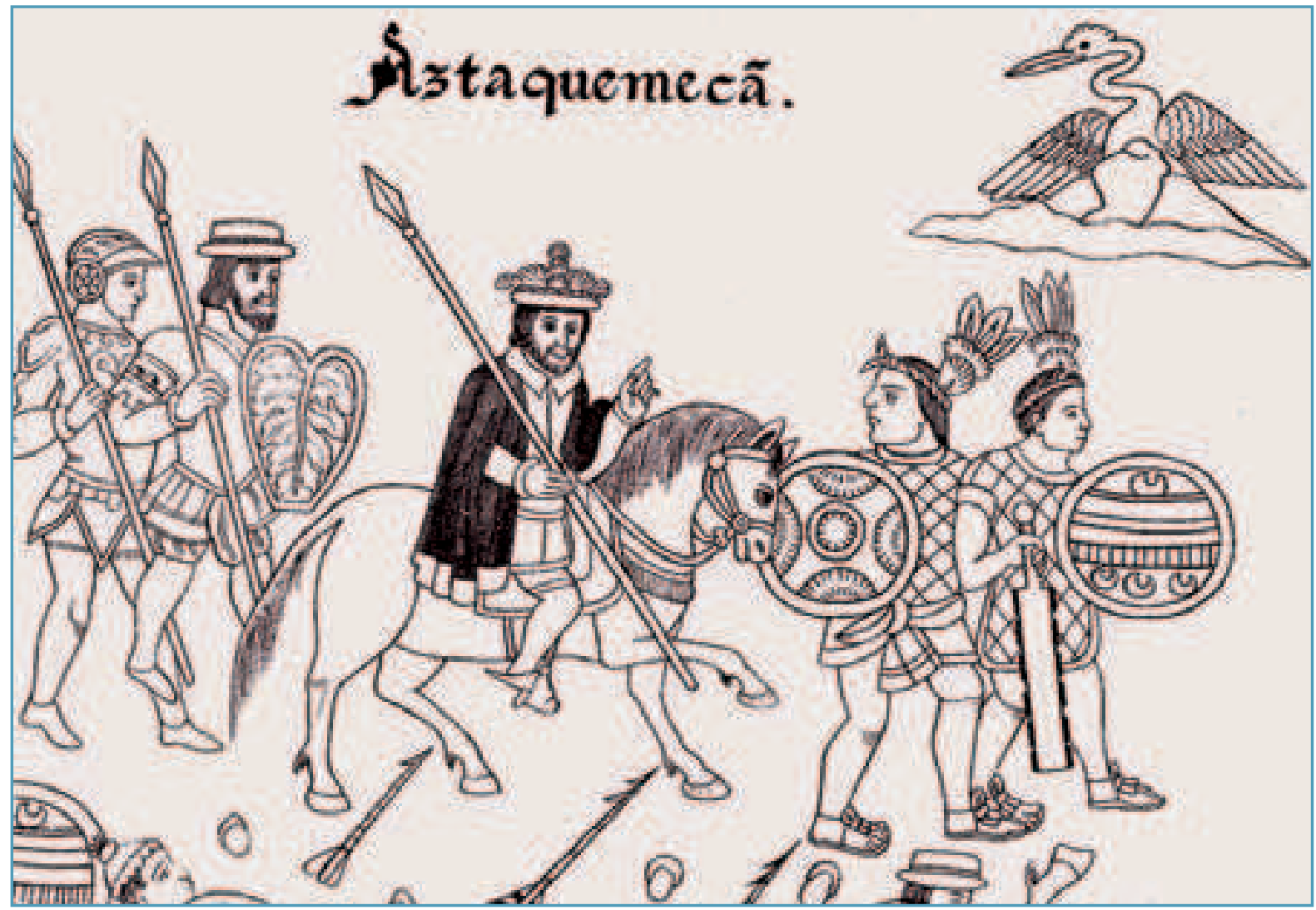

Abb. 5. Hernán Cortés mit indianischem Kopfschmuck (Lienzo de Tlaxcala, aus [16].

Auf die Eroberung Mexicos und Zentralmerikas durch Hernán Cortés (Abb. 5) und seine Nachfolger ${ }^{2}$ mit Pferden, Kanonen und Bluthunden wobei die spanischen Eroberer durch indigene Truppen unterstützt wurden - soll hier nicht näher eingegangen werden. Über die verheerenden Folgen für die einheimische Bevölkerung schrieb der Dominikaner Fray Bartolomé de las Casas ([1484-1566] in "Brevísima relación de la destrucción de las Indias"3, 1552, u.a.) sehr eindrücklich - wenn auch nicht sehr erfolgreich - an seinen Kaiser.

\footnotetext{
2 Siehe z.B. Xavier López Medellín (TenochtitlánMéxico) \& Felix Hinz (Köln). Berichte über Hernán Cortés.

http://www.motecuhzoma.de/start-deu.html

3 http://www.ciudadseva.com/textos/otros/brevisi.htm

4 im Internet einschaubar:

http://www.famsi.org/index.html

5 Wörterbuch Spanisch - Maya:

http://es.freelang.net/diccionario/maya.html
}

\section{Informationsquellen: Zentralamerikanische Literatur}

Der Säuberungswut der Inquisition entgingen nur wenige Schriftstücke, die z.T. als Kopie bis heute erhalten sind. Erhaltene Codices ${ }^{4}$ gibt es von den Maya $^{5}$ (nach ihrem Aufbewahrungsort benannt: Dresdner Codex, Pariser Codex [auch Codex Peresianus genannt], Madrider Codex, [auch Codex Tro-Cortesianus genannt] und den Grolier Codex), von den Azteken (vorspanische Codices: Codex Becker und Codex Cospi; aus der spanischen Kolonialepoche: Codex Florentino, Codex Mendoza, Codex Ixtlilxochitl), von den Mixteken (vorspanische Codices: Codex Zouche-Nuttall, Codex Vindobonensis Mexicanus). In medizinischen Angelegenheiten ist der meist Zitierte der Codex Florentinus; er stammt aus der spanischen Kolonialepoche. Er entstand unter der Leitung von Bernardino de Sahagún (1529-1590) als Teil der "Historia General de las Cosas de la Nueva España” mit Hilfe von
Azteken, um das erhaltene Wissen der Azteken-Kultur festzuhalten. Dieser Codex ist mit eine der wichtigsten Quellen über eine Zivilisation, die zu dem Zeitpunkt schon zerstört war. Er wird in der Bibliothek der Medicis in Florenz aufbewahrt und besteht aus 1846 Bildern. Die restlichen Codices befassen sich primär mit religiösen und astrologisch-mathematischen Problemen.

Popol Vuh ("Ratsbuch”) ist das heilige Buch der Quiché-Maya in Zentralamerika. Das Popol Vuh hat seinen Ursprung in uralten Überlieferungen, die im gesamten Maya-Kulturraum verbreitet waren und in Maya-Schrift aufgeschrieben wurden. Die Spanier (siehe Diego de Landa) verboten nach der Conquista die Verwendung der MayaSchrift und vernichteten die wertvollen Handschriften, soweit sie ihrer habhaft werden konnten, als “Teufelszeug”. Einigen Maya-Priestern gelang es jedoch, Abschriften alter Maya-Bücher anzufertigen, wobei sie auch schon lateinische Schrift verwendeten. Dieser Text lag unbeachtet in der Biblio- 
thek der Universidad de San Carlos in Guatemala-Stadt, bis er 1854 von Abbé Brasseur de Bourbourg und Carl Scherzer gefunden wurde (Abb. 6).

Es gibt einige Texte indigenen Ursprungs mit wichtigen Prophezeihungen, rituellen Mythen, aktuellen Ereignissen und einer chronologischen Synopsis der Geschichte, die die Nachfahren der Maya später während der Kolonialzeit auf Spanisch zusammentrugen. In den 100 Jahren nach der spanischen Eroberung werden einige dieser Manuskripte im Norden Yucatáns verfasst. Sie werden Bücher der Chilam Balam genannt. Chilam (oder Chilán) ist die Bezeichnung für einen Wahrsagepriester und Balam (Jaguar) steht für etwas Mysteriöses. Diese Bücher enthalten Berichte über Traditionen, Ritual- und Medizinalvorschriften sowie Ereignisse der Maya-Geschichte. Es muss von ihnen sehr viele gegeben haben, aber nur einige haben uns erreicht: Chilam Balam-Bücher von Maní, von Tizimín und von Chumayel.

\section{Der Kalender regiert das Leben}

Die Maya benutzten für rituelle und zivile Zwecke nebeneinander verschiedene Kalender, die auf einer Tageszählung im Zwanzigersystem beruhten: den rituellen Tzolkin-Kalender, den zivilen Haab-Kalender und die Lange Zählung, mit der längere Zeiträume erfasst werden konnten, die für Himmelsbeobachtungen und Astronomie eine grosse Rolle spielen. Die Kombinationen von Tzolkin- und HaabDaten wiederholen sich nach einer 52 Jahre dauernden Kalenderrunde. Da der Haab-Kalender 365 Tage und der Tzolkin-Kalender 260 umfasst, wiederholen sich alle 18'980 Tage (kleinstes gemeinsames Vielfaches von 365 und 260) oder 52 Haab-Jahre die Kombinationen von Haab- und Tzolkin-Daten. Dieser Zeitraum wird als Kalenderrunde bezeichnet, innerhalb derer eine Kombination aus Haab- und Tzolkin-Datum eindeutig ist. Ein solcher "Neustart" war für sie von grösster Bedeutung; entsprechend der zyklischen Geschichtsauffassung in Mittelamerika glaubte man, dass sich danach auch die historischen Ereignisse (im Wesentlichen) wiederholten. Nach
13 Bactun (= ca. 5000 Jahre) wird jeweils ein Weltuntergang erwartet. Der Azteken-Kalender besitzt grosse Ähnlichkeit mit dem älteren Maya-Kalender, zum Beispiel auch eine 52-jährige Kalenderrunde. Allerdings weichen die Datumsbezeichnungen voneinander ab. Der Azteken-Kalender verfügt ausserdem nicht über die strikt eingehaltene Lange-Zählung des Maya-Kalenders.

\section{Blutopfer und \\ Selbstverstümmelung}

Die Blutopferriten haben in den zentralamerikanischen Kulturen eine zentrale Bedeutung, um die Götter bei Laune zu halten, insbesondere an, gemäss Kalender, unheilvollen Tagen; z.B. galt Venus als ein besonders negativer ,Stern'. Grundsätzlich konnte Blut aus allen Körperteilen entnommen werden, bevorzugt waren jedoch die Leistengegend, die Wangen und Unterlippe und als besonders heilige Orte galten Zunge, Penis und Ohrläppchen. Die Blutentnahme aus der Leistengegend steht im Zusammenhang mit dem männlichen Ritus des Blutverstreuens, gelegentlich ist hierzu auch der Penis benutzt worden. Alle übrigen Organe wurden von

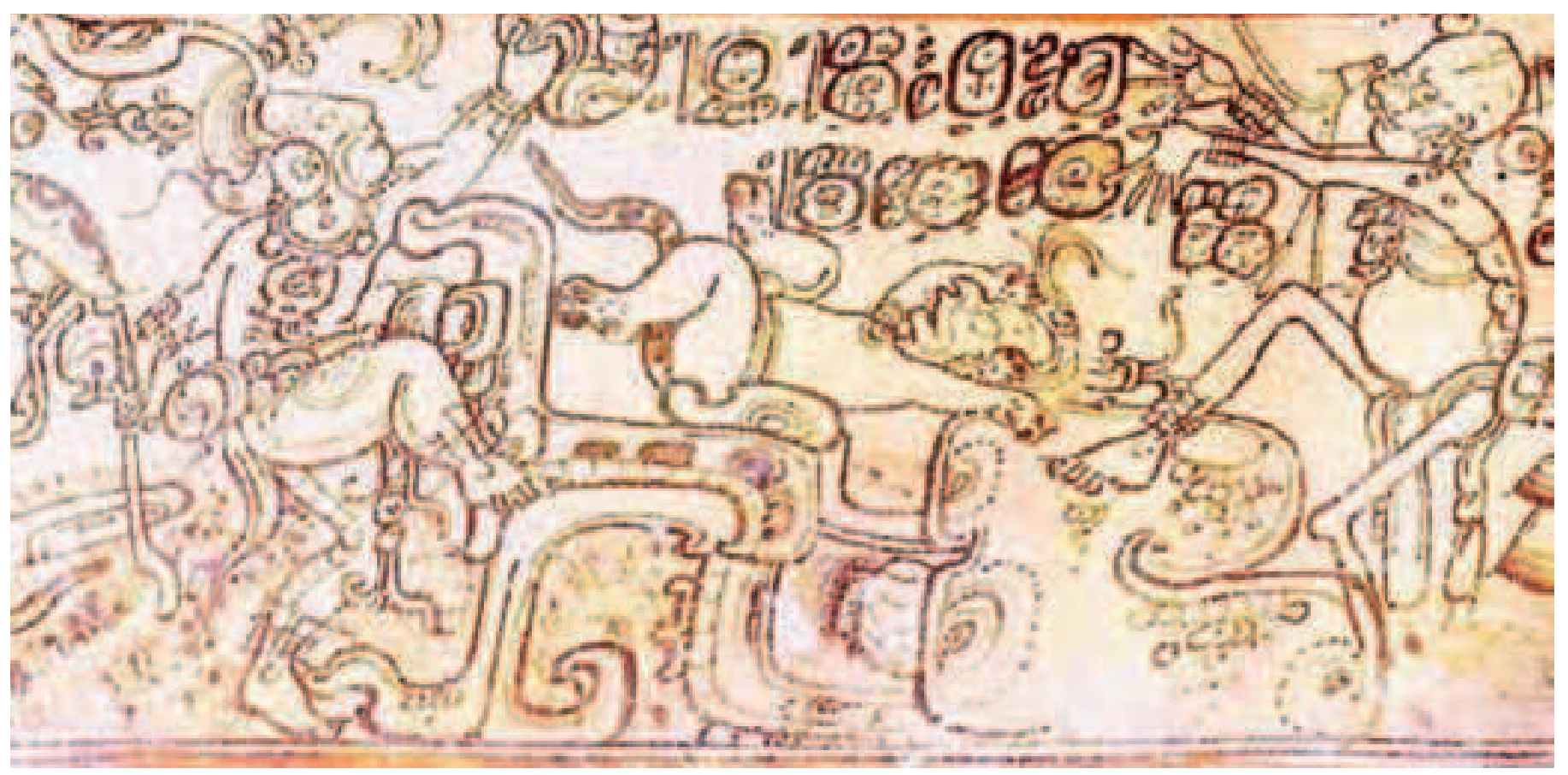

Abb. 6. Nach dem Popol Vuh: die Geburt von Hunahpú und seinem Bruder Ixbalanqué. Hunahpú - der Blasrohrjäger - und sein Bruder kämpften für die Armen und bestraften die Hochmütigen. Er dürfte auch für den unsichtbaren Hauptgott Hunab Ku stellvertretend dargestellt worden sein. 
Personen beiderlei Geschlechts benutzt. In der Relación de las cosas de Yucatán wird auch berichtet, dass beide Geschlechter sich runde Teile aus den Ohrläppchen schnitten oder die Männer sich gelegentlich die Vorhaut ihres Penis abrissen, (weshalb Chronisten glauben, dass bei den Maya die Beschneidung üblich sei). Die durch das Loch hindurch zu ziehende Schnur war etwa einen halben bis maximal einen Zentimeter dick und hatte wohl eine durchschnittliche Länge von ein paar Metern. Diego de Landa zufolge wurden durch den seitlich perforierten Penis auch Schnüre hindurch gezogen. Die Opfergänger bereiteten sich vor der Blutentnahme mit Tagen des Fastens, der Abstinenz und durch rituelle Dampfbäder auf den Ritus vor. Zur Blutentnahme stand den Maya, Azteken, usw., eine Reihe von verschiedenen Gegenständen zur Verfügung; gebräuchlich waren u.a. die Stacheln von Blättern der Agave, geschärfte und angespitzte Röhrenknochen; im Allgemeinen wurde jedoch ein dreiteiliges Instrumentarium, bestehend aus einer Obsidianlanzette, einer Feuersteinschneide und einem Rochenstachel (Dasyatis americana) bzw. einer Lanzette als Imitation desselben verwendet, die mit einem gesägten Rand versehen und im Lebendzustand der Tiere mit Gift gefüllt sind. Rochenstacheln wurden oft in Gräbern nahe der Beckenregion gefunden und befanden sich ursprünglich wohl in einem Beutel, der am Gürtel getragen wurde.

Die während des Opferns mit Blut getränkten Schnüre und Papierstreifen wurden während des Hindurchziehens durch die perforierten Körperteile in eine Opferschale oder einen Korb herabgelassen, gefüllt mit weiterem Opferpapier und weiteren Beigaben. Diese Beigaben wurden meistens mit Copal (Cupania belizensis) ${ }^{6}$

\footnotetext{
6 Dieser Baum wurde von den Maya benutzt, um Weihrauch zu gewinnen, den sie in ihren Zeremonien verwendeten. Das Holz ist rötlich und es wird nur für Bauten in ländlichen Gebieten und als Brennstoff verwendet. Der Copal findet keine kommerzielle Verwendung ausser zur Produktion von Weihrauch. Das Harz wird auch noch heute bei Festen und rituellen Opfergaben verbrannt. Die Maya glauben, dass es eine reinigende Funktion hat und sein dicker Rauch zum Himmel aufsteigt und dabei Botschaften an die Götter transportiert.
}

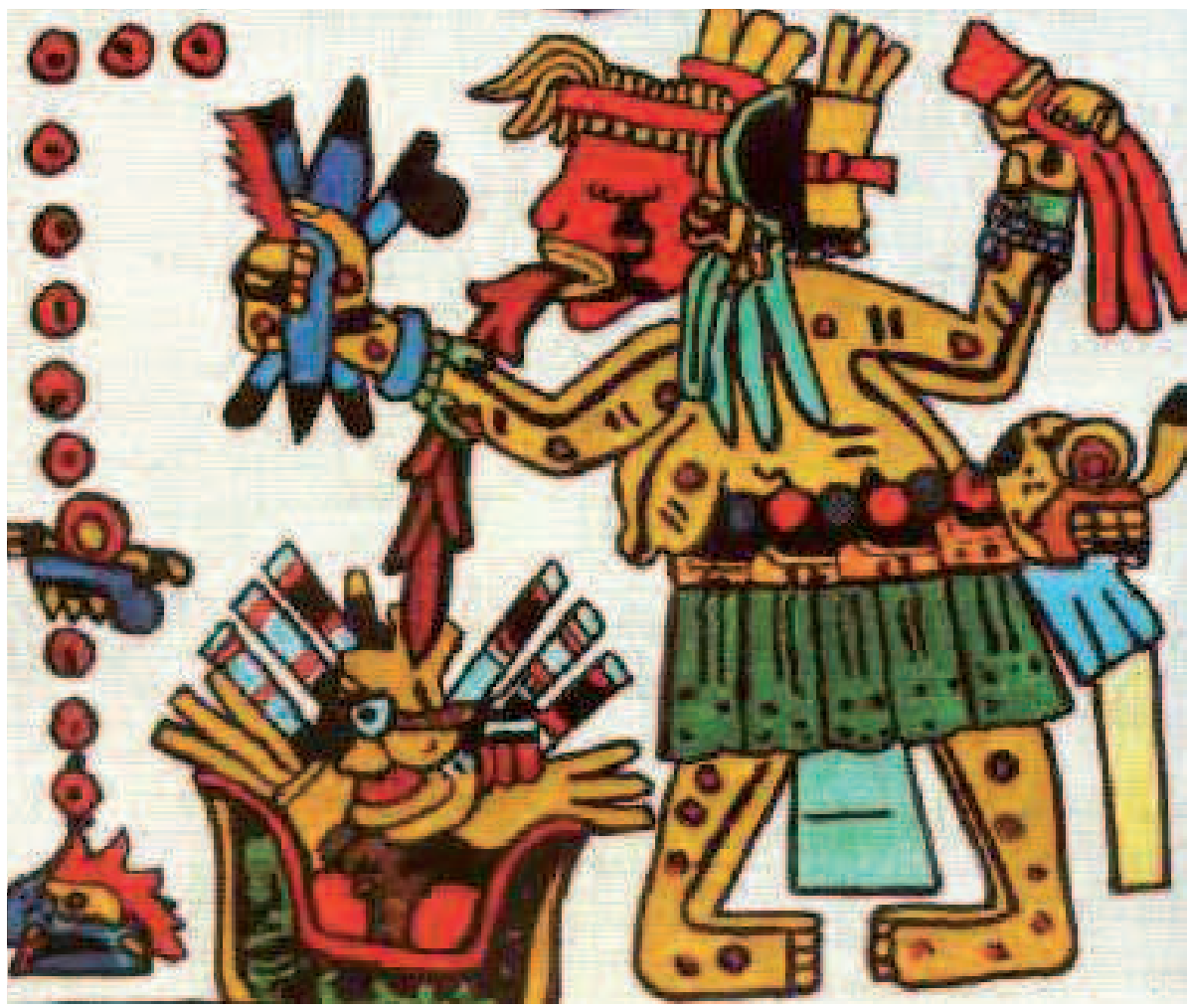

Abb. 7. Tlazolteotl (Aztek) konnte in Menschen eindringen und Konvulsionen auslösen. Hier dargestellt ist die Göttin selbst während einem Anfall; Schaum und Blut treten aus dem Mund, Tränen fliessen und die Füsse sind spastisch verdreht und ,verkrampft'.

in einer offenen Kohlepfanne verbrannt, die z.T. einen Aufsatz in Form einer Göttermaske (span. Incensarios) tragen konnte, wobei der Rauch durch Öffnungen durch den Mund und Augen entweichen konnte [17]

\section{Zwischen Magie und Medizin}

Die Gesundheit wird als das Resultat des Gleichgewichts der Wärme-KältePolarität betrachtet. Geht dieses Gleichgewicht verloren, so entsteht eine Krankheit-verursachende Störung in der Beziehung zwischen Körper und Kosmos. Der Nutzen der überlieferten Medizinal (ticiotl)-Bräuche ist meist nur ungenügend studiert worden, wobei allerdings z.T. auch schädliche Methoden und toxische Pflanzen benützt wurden (und zum Teil auch heute noch werden) [18]. Epilepsie hatte eine grosse magisch-religiöse Bedeutung wie ja in den meisten alten Kulturen und wurde mit einer der wichtigsten Gottheiten, der Tlazolteotl - Göttin der Lust und Erdmutter der Azteken - in

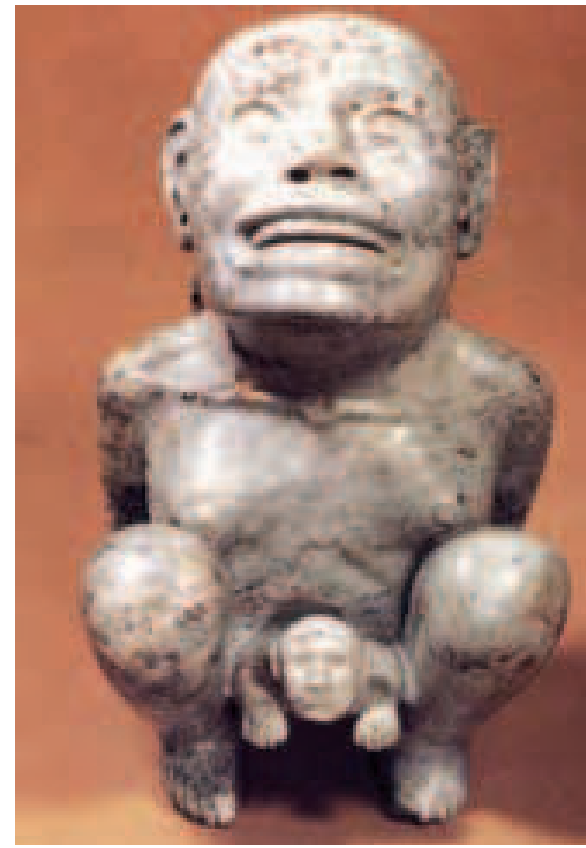

Abb. 8. Tlazolteotl - Erdmutter der Azteken gebärt den Maisgott.

Verbindung gebracht (Abb. 7 und 8). Die Behandlung beruhte primär auf magischen Mitteln, sekundär kamen Pflanzenpräparationen zum Einsatz [19]. 


\section{Rausch oder}

\section{metaphysisches Erlebnis?}

Zentralamerikanische Völker betrachteten den menschlichen Körper als Kern und vereinenden Körper des Kosmos, welcher von spezifischen übernatürlichen Kräften und Entitäten durchdrungen war. Der menschliche Körper würde progressiv aufgefüllt bei der Empfängnis, Geburt, erstem Kontakt mit dem Feuer, der Sonne und anderen bedeutenden Momenten des Lebens - mit Kräften, die in den himmlischen Sphären ihren Ursprung hatten, sowie mit heiligen Geschehnissen aus mythischer Zeit [20]. In dieser Konzeption liegen sie natürlich in totalem Widerspruch zu der jüdisch-christlich getrennten Dualität von Mensch und Gott.

Wie sah die Realität aus? Gemäss den Berichten der ,Conquistadores', und im Gegensatz zu den Azteken?, gab es kein Fest, bei dem die Maya sich nicht masslos betranken. Die Maya hatten keine stark alkoholhaltigen Getränke - vermutlich vorwiegend Balche, welches aus Wasser, Wildbienenhonig und Rinde des Balche-Baumes (Lonchocarpus longistylus) hergestellt wurde. Um den gewünschten Rausch zu erzielen, musste man Unmengen von diesem Balche trinken, was wiederum $\mathrm{zu}$ Erbrechen führte. Eine weitere Möglichkeit bestand darin, dieses mit Tabak, Blättern der Engelstrompete (Brugmansia spp.) und Stechapfelsamen (Datura spp.) zu vermischen. Dieses wohl nicht sehr schmackhafte Gemisch wurde dann auch vorwiegend mit einem - aus Kürbis und einem Tonrohr hergestellten - Klistier rektal verabreicht. Die Maya verwendeten ebenfalls das bufoteninhaltige Giftsekret der Ochsenkröte (Bufo marinus) und im Hochland - wie allgemein in den höheren Gegenden Mexicos - psilocybinhaltige Pilze (Tab. 2 und 3, Abb. 9). Sowohl Schamanen wie Gott-Könige bedienten sich dieser Mittel und Rituale, um Beistand

\footnotetext{
7 "Among the ancient Aztecs, getting drunk without incurring the wrath of society was a privilege of old age; drunkards could receive severe punishments, ranging from public disgrace to death by stoning or beating" (de Smet, Peter A.G.M, 1985. Ritual enemas and Snuffs in the Americas. Amsterdam: Centre for Latin American Research and Documentation)."
}

Tab. 2. Die Wirkung von Krötensekret, d.h. Bufotenin(e) (5-Hydroxy-Dimethyltryptamin und Derivate), Bufogenine und Bufotoxine

\section{Psychische Erscheinungen}

optische Illusionen und bildhafte Phänomene, insbesondere Farbeindrücke echte Halluzinationen unter Realitätsverlust

Selbstüberschätzung

Bewusstsein und Aufmerksamkeit vollständig mit den Erscheinungen ausgefüllt Eingeschränkte Auffassung \& Kritikfähigkeit

Euphorie

Logorhoe

Später Abkehrung von der Aussenwelt

Möglich: Auslösung psychotischer Zustände; Verwirrtheit

\section{Systemische Effekte}

schnell einsetzende vegetative Erregung: Gesichtsrötung

erweiterte Pupillen

Kopfschmerz

Schwindelgefühl

erhöhter Blutdruck und Puls

Anregung der Atmung

Zentralnervöse Erregung, zwanghafte

Motorik

Möglich: Auslösung von Herzrhythmusstörungen

Tab. 3. Von den Azteken und anderen Stämmen benützte Psychotrope Pflanzen [25]

$\begin{array}{ll}\text { Halluzinogene } & \text { Péyotl (Lophophora williamsii) } \\ & \begin{array}{l}\text { Teonanácatl } \\ \text { (Psilocybe mexicana, Psilocybe aztecorum) }\end{array} \\ \text { Induktoren von Trance-Zuständen } & \text { pipiltzintzintli (Salvia divinorum) } \\ \text { Induktoren von Kognitionsstörung } & \text { ololiuhqui (Turbina corymbosa) } \\ \text { Induktoren von Delirium } & \text { Tolohuaxíhuitl (Datura stramonium) } \\ \text { Stimulantien } & \text { Maya: xigar (Nicotiana tabacum) }\end{array}$

bei Ahnen und Göttern zu holen [21]. Das gemeinsame Erleben religiöser und mythischer Themen im Rausch und deren Betonung und Prägung durch die schamanische Interpretation sollte auch zur Stabilisierung des jeweiligen Weltbildes beitragen. Der visionäre Gehalt Halluzinogen-induzierter Ekstasen dient in allen Fällen kulturell spezifizierten Zielen wie Divination, Opferungen, Regenzauber und der Diagnose von Krankheiten, wobei der Schamane als eine Art Führer fungiert. Die Ekstase ist durch einen Wechsel von der Alltagswelt in die spirituelle Welt gekennzeichnet. Für den Kontakt mit der spirituellen Welt bedarf es physischer und psychischer Vorbereitungen. Diese und die zu schaffenden Rahmenbedingungen beziehen sich auf das Sammeln, die Zubereitung und die Einnahme der Drogen, ausserdem auf die dazugehörenden oder zu schaffenden Umstände, wie Anlass, Wahl des Ein- nahmeortes und des Zeitpunktes. Dies gilt ebenso für die nicht-kollektiven Zeremonien, an denen neben dem Schamanen auch der zu behandelnde Patient oder andere Stammesmitglieder das Halluzinogen einnehmen. Die Institution der Halluzinogen-Einnahme bzw. der Trance wird in allen Beispielen durch Mythen und Sagen unterstützt. In ihnen wird dargelegt, wie das Halluzinogen entstand und auf welche Weise die Menschen den Umgang mit ihm lernten. Sie handeln auch von der Kommunikation zwischen Menschen und Göttern im Zusammenhang mit dem Halluzinogen und bieten somit Information über die Inhalte der Ekstase [22]. Häufig werden Zwerge als Begleiter der Herrscher und Boten der Unterwelt, welche über Höhlen und Erdlöcher mit der bekannten Welt verbunden war, dargestellt [23].

WADE DAVIS beschrieb seine Erfahrung nach dem Rauchen von Bufotenin (Bufo alvarius): 
"Shortly after inhalation, I experienced warm flushing sensations, a sense of wonder and well-being, strong auditory hallucinations, which included an insect-cicada sound that ran across my mind and seemed to link my body to the earth. Though I was indoors, there was a sense of the feel of the earth, the dry desert soil passing through my fingers, the stars at midday, the scent of cactus and sage, the feel of dry leaves through hands. Strong visual hallucinations in orblike brilliance, diamond patterns that undulated across my visual field. The experience was in every sense pleasant, with no disturbing physical symptoms, no nausea, perhaps a slight sense of increased heart rate [24]."

Die ersten schriftlichen Zeugnisse vom Gebrauch berauschender Pilze bei festlichen Anlässen oder im Rahmen von religiösen Zeremonien und magischen Heilpraktiken findet man schon bei den spanischen Chronisten und Naturalisten aus dem 16. Jahrhundert, die bald nach der Eroberung von Mexiko durch Hernan Cortés ins Land kamen. Der Franziskanerfrater Bernardino de Sahagún erwähnt die Zauberpilze an mehreren Stellen seiner Schriften, wo er ihre Wirkungen und ihren Gebrauch beschreibt. "Bei der festlichen Zusammenkunft zu der Zeit, wenn die Flöten geblasen werden, assen sie Pilze. Sie nahmen keine andere Nahrung ein; sie tranken die ganze Nacht nur Schokolade. Sie assen die Pilze zusammen mit Honig. Als die Pilze zu wirken begannen, wurde getanzt und geweint ... Einige sahen in ihren Visionen, wie sie im Krieg starben ..., einige, wie sie von wilden Tieren aufgefressen wurden ..., einige, wie sie wohlhabend wurden und Sklaven kaufen konnten ..., einige, wie sie Ehebruch begingen und wie sie dann gesteinigt und ihre Schädel eingeschlagen wurden ..., einige, wie sie im Wasser ertranken ..., einige, wie sie im Tod die Ruhe fanden, einige, wie sie vom Hausdach zu Tode fielen ... Alle diese Dinge sahen sie. Als die Wirkung der Pilze nachliess, sassen sie zusammen und erzählten einander, was sie

8 Ein Wörterbuch Spanisch - Nahuatl:

http://www.tula.gob.mx/congreso/Espaol-Nhuatl.html in ihren Visionen gesehen hatten.» Auf Nahuatl (Azteken ${ }^{8}$ ) wurden diese Pilze als "Teonanacatl I" bezeichnet, was mit "göttlicher Pilz" übersetzt werden kann. In Guatemala, in El Salvador und in den anschliessenden gebirgigen Gegenden Mexikos sind so genannte "Pilzsteine" gefunden worden. Es sind dies Steinplastiken von der Form eines Hutpilzes, in dessen Stiel das Antlitz oder die Gestalt eines Gottes oder tierartigen Dämons gemeisselt ist. Die berauschenden, Visionen und Halluzinationen erzeugenden Wirkungen dieser Pilze erschienen den christlichen Missionaren als Teufelswerk. Sie versuchten daher mit allen Mitteln, deren Gebrauch auszurotten. Das gelang ihnen aber nur teilweise, denn die Indianer verwendeten den heiligen Pilz „Teonanacatl” im Geheimen bis in unsere Tage weiter (aus [26]).

Psilocybin (Klinische Studie mit $30 \mathrm{mg}$ pro $70 \mathrm{~kg} \mathrm{KG}, 30$ Probanden, doppelblind vs. Ritalin als Placebo) hat in letzter Zeit wieder vermehrt das Interesse der Forschung im Zusammenhang mit dem Auslösen von mystisch-religiösen Erlebnissen erweckt [27]. Rund zwei Drittel der Freiwilligen beschrieben Effekte, welche die Kriterien einer "vollständigen mystischen Erfahrung” nach etablierten psychologischen Massstäben erfüllten; sie stuften die Erfahrung unter den fünf bedeutsamsten spirituellen Erlebnissen ihres Lebens ein - für viele rangierte die Bedeutung des Ereignisses auf vergleichbarer Ebene mit der Geburt des ersten Kindes oder dem Tod eines Elternteils. Ein Drittel der Teilnehmer berichtete aber auch von Angstattacken und kurzen Anflügen von Paranoia. Noch zwei Monate später berichteten fast achtzig Prozent der Probanden von grösserer Zufriedenheit oder Wohlgefühl sowie einem anhaltenden positiven Effekt auf Stimmung und Verhalten.

\section{Medizinalpflanzen}

Kakao: Der Kakao diente nicht nur als Genussmittel, sondern wurde auch als Zahlungsmittel verwendet. Der Wert der Kakaobohne zur Zeit des Aztekenreiches ist zwar unbekannt, aber die Währung der Kakaobohnen bestand auch unter spanischer Herrschaft fort
(z.B. im Jahre 1545 hatte ein Truthahn einen Wert von 200 Kakaobohnen; ein Hase oder Waldkaninchen war je 100 Kakaobohnen wert; eine grosse Tomate (Aztek.: “Tomatl”, “Tumatl” oder "Xitomatl”) entsprach dem Wert einer Kakaobohne [28]. Seit ca. 250 v. Chr. kultivierten die Maya - wie vor ihnen die Olmeken - die aus Südamerika stammende Kakaopflanze. Die Maya tauschten Kakaobohnen gegen andere für sie nützliche Gegenstände; der Tauschhandel umfasste ganz Mittelamerika bis nach Kolumbien. Auch mit den Azteken, die ab 1200 n. Chr. im Hochland von Mexiko auftauchten, wurde ein reger Handel betrieben. Der Kakao spielte im kulturellen Leben der Azteken eine grosse Rolle. Da in ihrem Reich der empfindliche Kakaobaum aus klimatischen Gründen nicht wuchs, waren die Azteken auf den Handel mit den Maya angewiesen. Der Kakao wurde schon früh als Medizin genutzt: Man setzte ihn gegen Fieber, Vergiftungen, Schlangenbisse und zur Behandlung (desinfizieren?) von Wunden ein.

Auch die Depression, die ihren Ursprung im Herzen (yollotl) hatte, wurde u.a. mit Kakao (Xocoatl) behandelt; im weiteren wurden eine Diät mit Fisch, Hasen und Hühnchen sowie magischreligiöse Lebensstiländerungen empfohlen [29].

Eine kurze Beschreibung der Arbeitsschritte zur Herstellung von Schokolade überlieferte FRAY BERNANDINO DE SAHAGún [30]: "Sie mahlt die Kakaobohnen [Xocoatl]; sie zerstampft, bricht und zerkleinert sie zu Pulver. Sie sortiert sie aus, verliest und trennt sie. Sie durchtränkt sie, durchfeuchtet sie, weicht sie ein. Sie fügt sparsam, zurückhaltend Wasser hinzu; sie reichert es mit Kohlensäure(?) an, filtert es, siebt es, schüttet es hin und her, bringt es zum Sprudeln; sie lässt es eine Krone bilden, stellt Schaum her; sie entfernt die Krone, lässt es dickflüssig werden und trocknen, giesst Wasser dazu, rührt Wasser hinein."

Einfachen Schokoladengetränken wurden gemahlener Mais und andere gemahlene Körner (z. B. Samenkörner des Woll- oder Seidenbaumwollbaums) zugegeben. Überall in Mittelamerika war die Zugabe von zerriebenem Chili 

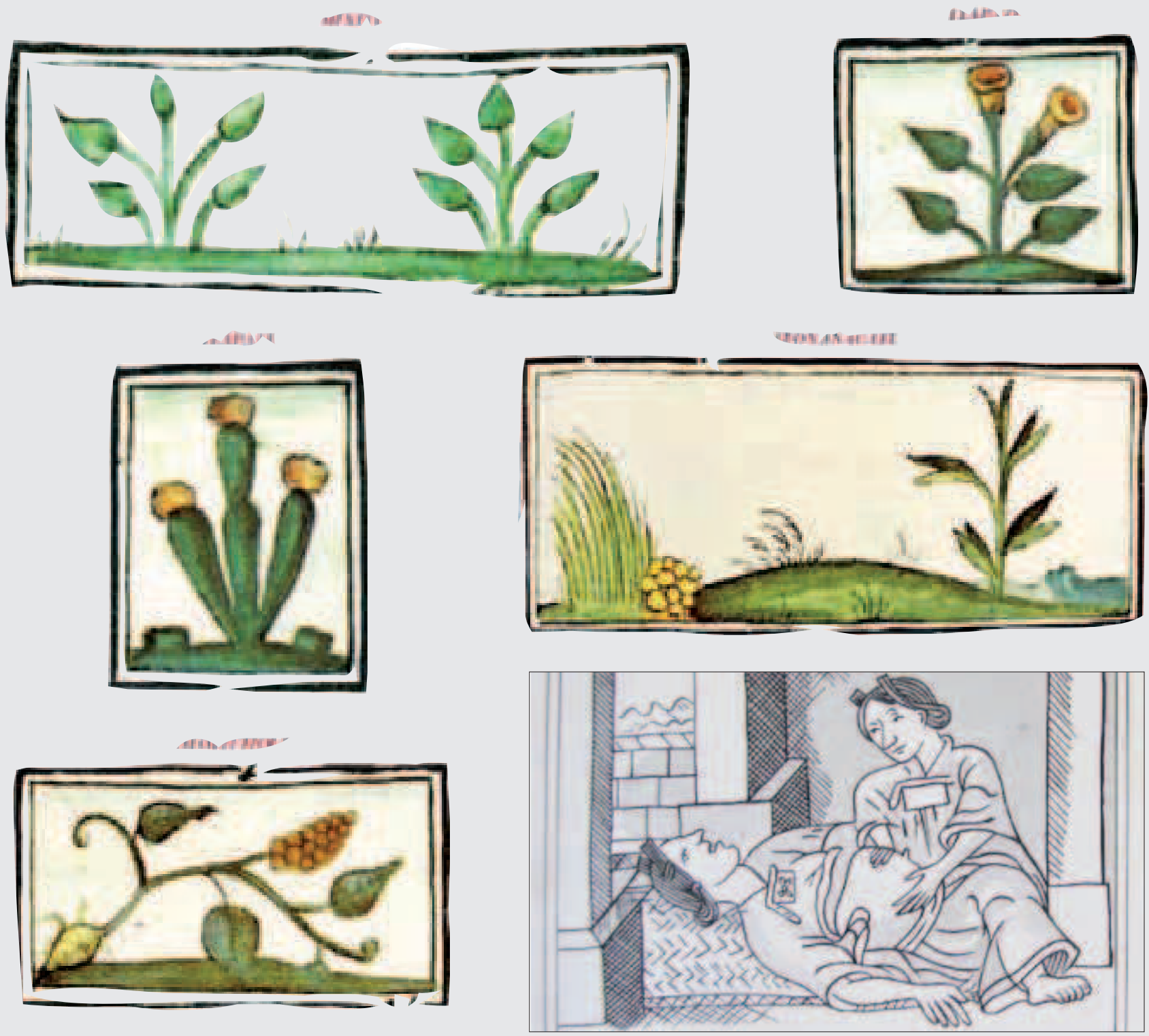

Abb. 9. Ausschnitte aus dem Codex Florentino: psychoaktive Pflanzen; eine Geburt.

sehr beliebt. In der präkolumbianischen Medizin galt Kakao auch als magische und heilende Pflanze. (Dunkle Schokolade hat einen hohen Gehalt an Flavonoiden bez. Antioxidanzien). Moderne Studien zeigten, dass Kakao (bez. Schokolade) den Blutdruck senkt und die kardiovaskuläre Mortalität reduziert [31].

\section{Weitere Medizinalpflanzen}

Chilischoten (Capsicum annuum, Capsicum baccatum, Capsicum chinese, Capsicum frutescens und Capsicum pubescens) wurden nicht nur als Ge- würz, sondern auch zur Behandlung verschiedener (wahrscheinlich infektiöser) Leiden eingesetzt. Eine moderne Studie [32] hat gezeigt, dass kalt gepresste oder auch warm extrahierte Extrakte antimikrobielle Eigenschaften besitzen (Bacillus cereus, Bacillus subtilis, Clostridium sporogenes, Clostridium tetani und Streptococcus pyogenes). Extern appliziertes Capsaicin (Capsicum Frutescens) ist ebenfalls zur Linderung von Rückenschmerzen wirksam [33]. Capsaicin, der scharfe Stoff des roten Paprikas, stimuliert sensible Nerven in der Magenschleim- haut, steigert dadurch die Magendurchblutung und verstärkt andere lokale Schutzmechanismen [34]. Die Selektivität seiner Wirkung macht Capsaicin zu einem wichtigen pharmakologischen Werkzeug in der Erforschung von schmerzleitenden und hitzeempfindlichen Nervenfasern.

Der Xocotl (Span. Jocote, Matasano; Casimiroa tetrameria MILLSP. (Rutaceae)). Diese Frucht wirkt angeblich hypnotisch, wie auch Cochiztzapotl des Iztaczapotl (Span. Zapote blanco; Casimiroa edulis). Die Arzneipflanze wird bei den Maya auf der mexikani- 
schen Halbinsel Yucatán traditionell bei gastrointestinalen Beschwerden (Diarrhoe) verwendet. Eine Teezubereitung der Pflanze nach Art der Maya erwies sich in ,in vitro' als biologisch aktiv: Am isolierten Meerschweinchen-Ileum wurden die durch Histamin ausgelösten Kontraktionen um bis zu $88 \%$ inhibiert. Ausserdem wird das Wachstum von Helicobacter pylori durch den Rohextrakt gehemmt [35].

Cihuapatli (Montanoa tomentosa) wurde von den Azteken zur Induktion von Geburtswehen eingesetzt; tierexperimentelle Studien [36] haben eine oxytocinartige Wirkung gezeigt (und es ist, zumindest bei der Ratte, ein Aphrodisiakum).

Der Agavensaft wurde sowohl für rituelle Zwecke, zu Pulque fermentiert, und für medizinische Zwecke gegen Schlangenbisse, Blähungen, Verstopfung, Fieber, Hautkrankheiten, Zahnschmerzen usw. eingesetzt. (Aus den hartfaserigen Blättern wurden Körbe geflochten und Sandalen hergestellt. Die Creme, die sich unter der Haut der Blätter und den Wurzeln befand, wurde als Seife benutzt).

Einige weitere gut bekannte Medizinalpflanzen aus Zentralamerika sind:

- Cáscara sagrada oder jalapa (Convolvulus jalapa) wird als Laxans benützt.

- Mais (Zea mays), wobei besonders die fadenförmigen Maisgriffel der weiblichen Blüten bei Leiden der Harnwege angewendet werden. Sie gelten als leicht diuretisch wirkend.

- Brechwurzel (Psychotria emética, Psychotria ipecacuanha): Die Wurzel wird verwendet, um ein starkes Brechmittel herzustellen; Emetin ist ein Amoebizid.

- Guayacán (Guayacum officinale, Guaiacum sanctum): Linderung bei Erkältungen, schweisstreibend, sollte auch gegen (syphilitsche?) Beulen wirksam sein (Span.: palo de bubas).

- Zarzaparrilla (Span. palo de la vida; smilax sp) schweisstreibend, gegen Gicht, Magentonikum, und wurde sogar gegen Syphillis eingesetzt.

- Perubalsam (Myroxylon peruiferum)

9 http://www.napraler.org/ stammt eigentlich aus Mexico (Azt.: huitziloxitl) und wurde extern zur Wundbehandlung und gegen Parasiten eingesetzt. Ähnliche Anwendungen fand der Bálsamo de Tolú (Myroxylom balsamum oder Toluiferum balsamum).

- Maiapfel (Podophyllum peltatum), Abführmittel; wird extern noch heute gegen Warzen eingesetzt (Podophyllotoxin).

- Gänsefuss (Chenopodium ambrosioides var. antihelminticum) wurde als Wurmmittel verwendet.

Die spezifische Behandlung konnte dann doch etwas eigenartig sein; so wurden z.B. traumatische Brustverletzungen mit Aderlass behandelt, wobei der Patient einen Trank aus warmem Harn und gemahlenen Eidechsen erhielt [37].

\section{Schlussfolgerung}

Wir hoffen, mit dieser kleinen zweiteiligen Exkursion in die zentralamerikanischen Kulturen und ihre Bevölkerungen einen Eindruck dieser faszinierenden, wenn auch brutalen Welt vermittelt $\mathrm{zu}$ haben. Nicht zu vergessen ist, dass, trotz punktuell hohen Entwicklungsstandards es sich um SpätNeolithische Kulturen handelte. Dennoch liefert uns der Erfahrungsschatz aus dieser Region immer wieder neue pharmakologische Ansätze So wurde anhand der Beobachtungen eines mexikanischen Zahnarztes das Affinin (spilanthol), ein stark lokalanästhetisch wirkendes 2,6,8-Decatrienisobutylamid, in der Heliopsis longipes (A. Gray) identifiziert [38]. Ethnomedizinische Informationen werden weltweit gesammelt und in verschiedenen Datenbanken wie z.B. Napralert ${ }^{9}$ zu- $^{-}$ gänglich gemacht.

\section{Danksagung und Angaben zur finanziellen Unterstützung}

An dieser Stelle möchten wir allen danken, die uns bei der Erstellung dieser Arbeit unterstützt haben. Unser ganz besonderer Dank geht an Frau Ruth Casanova, Zürich, für die sorgfältige Durchsicht des Manuskripts.

Diese Arbeit erhielt keine finanzielle Unterstützung.

\section{Literatur}

1. Brignoli R, Saller R: Medizin in (Latein)Amerika vor der Conquista. Teil I: Allgemeines und Andenregion. Schweiz. Zschr. GanzheitsMedizin 2007;19(1):48-55.

2. Brönnimann S: - Grossräumige Klimaschwankungen - WS 04/05

http://www.iac.ethz.ch/people/stefanbr/teach ing/vorlesung1/klimaschwankungen_42.pdf

3. Zollikofer R: Der Osten meines Heimatlandes im Hungerjahre 1817, St. Gallen 1818, Band l; zit. nach Hardegger Joseph et al, Das Werden der modernen Schweiz, Band 1, Basel und Luzern, 11986, S. $49 f$ zitiert in http://technik.geschichte-schweiz.ch/industrialisierung-schweiz.html

4. Lange Frederick W. Review of "Mountains of Fire, Lands That Shake: Earthquakes and Volcanic Eruptions in the Historic Past of Central America (1505-1899) by Lawrence $\mathrm{H}$. Feldman" Latin American Antiquity, Vol. 7, No. 1 (Mar., 1996), pp. 84-86, doi:10.2307/ 3537019

5. Kovach RL: Early Earthquakes of the Americas: Cambridge University Press, Cambridge, 2004, 268 pp, ISBN 0:521-82489-3

6. deMenocal PB: Cultural Responses to Climate Change During the Late Holocene. SClENCE 2001;292:667-773

7. Schurr TG, Ballinger SW, Gan YY, Hodge JA Merriwether DA, Lawrence DN, Knowler WC, Weiss KM, and Wallace DC: Amerindian mitochondrial DNAs have rare Asian mutations at high frequencies, suggesting they derived from four primary maternal lineages. Am J Hum Genet. 1990 March;46(3):613-623.

8. Merriwether DA (Reported by Sheri Fink): Peopling the New World, A Mitochondrial View. The New Yor Academy of Sciences Posted Dec 1, 2004

http://www.nyas.org/ebrief/miniEB.asp?ebrie $\mathrm{fID}=357 \#$

9. Merriwether DA, Hall WW, Vahlne A, Ferrell RE: $m$ tDNA variation indicates Mongolia may have been the source for the founding population for the New World. Am J Hum Genet. 1996 Jul;59(1):204-12.

10. Vieira AR, Karras JC, Orioli IM, Castilla EE, JC Murray: Genetic origins in a South American clefting population. Clinical Genetics Vol. 62, Issue 6, Page 458, December 2002.

11. Vargas-Alarcon G, Hernandez-Pacheco G, Rodriguez-Perez JM, Perez-Hernandez N, et al.: Angiotensin-Converting Enzyme Gene (ACE) Insertion/Deletion Polymorphism in Mexican Populations. Human Biology, Dec 2003.

http://findarticles.com/p/articles/mi_qa3659/i s_200312/ai_n9310264/pg_3

12. Vasconcelos Palacios G: La Primera Escuela de Medicina en Mesoamérica. Rev. Mex. Anest. 1985:8:143-149.

http://www.anestesia.com.mx/art33.html\#16

13. Williams JS and White ChD: Dental modification in the postclassic population from Lamanai, Belize. Ancient Mesoamerica 2006; 17:139-151.

14. Tiesler V, MS, PhD, Salomón MR, DDS, and Oliva I: Endodontics: Decoration Techniques in Ancient Mexico - A Study of Dental Surfaces Using Radiography and S.E.M. Oral Health, September 2002.

15. Tiesler Blos V.: Cranial Surgery in Ancient Mesoamerica 2003. www.mesoweb.com/features/tiesler/Cranial. pdf

16. Hinz : Der Wandel im Selbstverständnis des Hernán Cortés durch die Conquista. http://www.motecuhzoma.de/wandel.html

17. Gronemeyer S: Tranceerzeugung in den 
Selbstkasteiungsriten der Maya. Medizinische Grundlagen und Auswirkungen. Rheinische Friedrich-Wilhelms-Universität Bonn. Seminar für Völkerkunde. Halver, im Herbst 1999.

18. Kufer J, Forther H, Poll E, Heinrich M: Historical and modern medicinal plant uses - the example of the Ch'orti' Maya and Ladinos in Eastern Guatemala. J Pharm Pharmacol (2005 Sep) 57(9):1127-52.

19. Elferink JG: Epilepsy and its treatment in the ancient cultures of America. Epilepsia (1999 Jul) 40(7):1041-6.

20. Blainey M: Theses: Evidence for Ritual Use of Entheogens in Ancient Mesoamerica and the Implications for the Approach to Religion and Worldview. 2005.

http://www.wayeb.org/download/theses/blai ney_2005.pd

21. Grube N: Rausch \& Ekstase. In: Maya - Gottkönige im Regenwald (hg. von Nikolai Grube Köln: Könemann), 2000, S. 294-295

22. Rosenbohm A: Halluzinogene Drogen im Schamanismus. Mythos und Ritual im kulturellen Vergleich. Berlin: Reimer Verlag 1991. 178 S. (Marburger Studien zur Völkerkunde 8). ISBN 3-496-00401-0

23. Prager Ch: Hofzwerge in Maya - Gottkönige im Regenwald (hg. von Nikolai Grube Köln: Könemann), 2000, 278-279.

24. Davis W \& Weil A: 1992 Identity of a New World Psychoactive Toad. Ancient Mesoamerica 3:51-59. In Blainey M. Theses: Evidence for Ritual Use of Entheogens in Ancient Mesoamerica and the Implications for the Approach to Religion and Worldview. 2005. http://www.wayeb.org/download/theses/blainey_2005.pdf

25. Díaz JL. Alucininógenos prehispánicos: las plantas mágicas y la conciencia visionaria. Arqueología Mexicana 2003;10(59):18-25.

26. Hofmann A: LSD - mein Sorgenkind. Die Entdeckung einer "Wunderdroge" 9. Auflage Januar 2001. Deutscher Taschenbuch Verlag $\mathrm{GmbH} \&$ Co. KG, München. Www.dtv.de ISBN 3-423-36135-2

27. Griffiths RR, Richards WA, McCann U, Jesse $R$ : Psilocybin can occasion mystical-type experiences having substantial and sustained personal meaning and spiritual significance. Psychopharmacology (Berl). 2006 Aug;187(3): 268-83; discussion 284-92. Epub 2006, Jul 7.

28. Anderson AJO, Berdan F, Lockhart J, "Beyond the Codices: The Nahua View of Colonial Mexico", Berkeley und Los Angeles, University of California Press 1976.

29. Rodriguez-Landa JF, Pulido-Criollo F, Saavedra M: Depression in Precolumbian Mesoamerican medicine. La depresion en la medicina mesoamericana precolombina. Rev Neurol (2007 Mar 16-31);44(6):375-80.

30. Sahagún, Fray Bernardino de: "General History of the Things of New Spain". Übersetzung aus dem Nahuatl durch Arthur J.O. Anderson und Charles E. Dibble, 12 Bde. Santa Fee, School of American Research and University of Utah 1950-1959.

31. Buijsse B, Feskens EJM, Kok FJ, Kromhout D: The Zutphen Elderly Study. Cocoa Intake, Blood Pressure, and Cardiovascular Mortality. Arch Intern Med. 2006;166:411-417.

32. Cichewicz $\mathrm{RH}$, Thorpe PA: The antimicrobial properties of chile peppers (Capsicum species) and their uses in Mayan medicine. $J$ Ethnopharmacol 1996;52(2):61-70.

33. Gagnier JJ, vanTulder M, Berman B, Bombardier C: Herbal medicine for low back pain. The Cochrane Database of Systematic Reviews 2006, Issue 2. Art. No.: CD004504. pub3.

DOI: 10.1002/14651858.CD004504.pub3.
34. Holzer P: TRPV1 and the gut: from a tasty receptor for a painful vanilloid to a key player in hyperalgesia. European Journal of Pharmacology 2004;500:231-241.

35. Bilkis $\mathrm{H}$ : Isolierung gastrointestinal wirksamer Inhaltsstoffe aus Casimiroa tetrameria MILLSP., einer yukatekischen Arzneipflanze der Maya (Mexiko).

http://www.freidok.uni-freiburg.de/volltexte/ 442/

36. Carro-Juarez M., Lobaton I., Benitez O., Espiritu A: Pro-ejaculatory effect of the aqueous crude extract of cihuapatli (Montanoa tomentosa) in spinal male rats. Journal of Ethnopharmacology 2006;106(1):111-116.

37. Ortiz de Montellano B: Medicina y salud en Mesoamérica. Arqueología mexicana 2005; 13(74):32-37. ISSN 0188-8218

38. Fabricant DS and Farnsworth NR: The Value of Plants Used in Traditional Medicine for Drug Discovery. Environ Health Perspect 2001;109(suppl 1):69-75

\section{Korrespondenzadresse:}

Prof. Dr. med. Reinhard Saller

UniversitätsSpital Zürich

Dep. für Innere Medizin

Institut für Naturheilkunde

Rämistrasse 100, CH-8091 Zürich

reinhard.saller@usz.ch 